\title{
Another Route to Lunar Magnetization
}

Although the Moon has no measurable internal magnetic field at present, the discovery that some of the returned lunar samples contain stable magnetizations is usually taken as evidence for the existence of a lunar field sometime in the past. The Apollo 11 and Apollo 12 samples, for example, were dated at about 3.6 and 3.3 billion years, respectively, which suggests that a lunar field, if not intermittent, existed for a period of at least 300 million years. The further implication of this is that for at least the same period of time the Moon must have possessed a fluid conducting core. The presence of magnetizations in lunar rocks is thus assumed to have important consequences for the history of the Moon's structure.

But what if the lunar rock magnetizations were acquired in some process essentially external to the Moon? In one sense this possibility has long been recognized. The lunar rocks were carefully tested upon their return to ensure that the magnetizations were not comparatively unstable components acquired during, or subsequent to, their return to Earth. In fact, the magnetizations were extremely stable, and this gave confidence that they were thermoremanences acquired in a lunar field. Now, however, Butler and Cox (Science, 172, $939 ;$ 1971) have discovered a mechanism for producing magnetic remanence in lunar samples which may give very stable magnetizations without requiring the existence of a lunar field. This disturbing discovery must inevitably throw doubt on the whole chain of thought leading to the supposition of an ancient lunar core.

The new mechanism involves the conversion by neutron irradiation of a magnetically soft isothermal remanence to a remanence with a higher coercive force. For their experiments, Butler and Cox selected two metals with compositions similar to those found in meteorites and lunar samples-iron machined from a puron rod and annealed in hydrogen gas up to $1,200^{\circ} \mathrm{C}$, and kamacite made by alloying puron and electrolytic nickel to a final nickel composition of five per cent by weight and annealed in hydrogen up to $1,000^{\circ} \mathrm{C}$. Following the measurement of bulk coercivity for both materials and a full demagnetization curve for the saturation isothermal remanence (IRM) in a sample of iron, all samples were exposed to a total fluence of $3 \times 10^{17}$ neutrons per square centimetre produced by placing them in a reactor core for three hours. As a result of the irradiation the bulk coercivities of the iron and kamacite were increased by twenty-one per cent and sixteen per cent respectively. Measurement of the a.f. demagnetization curve for irradiated saturation IRM in iron showed, first, that the radiation had not destroyed the IRM and, second, that the coercive force of the IRM had increased. The assumed theory behind these results is that the irradiation produces lattice defects and associated stress fields which pin domain walls in the materials by magnetostrictive interaction. That such pinning is capable of giving large changes in coercive force spectra has, in fact, already been amply demonstrated for industrially important ferromagnetic materials.

But could such processes take place in real meteorites and lunar samples? Butler and Cox evidently believe they could - through cosmic irradiation. More than eighty per cent of the cosmic ray flux is made up of protons with average energies of over $4 \mathrm{GeV}$, which react with atomic nuclei to produce neutrons. A recent investigation by Eugster et al. (J. Geophys. Res., 75, 2753; 1970) of the isotopic composition of gadolinium in the Norton County aubrite indicated isotopic abundances equivalent to formation by a neutron fluence of $6 \times 10^{5}$ neutrons per square centimetre integrated over 240 million years. About one half of these neutrons had energies of several million electron volts. By comparison, about ten per cent of the fluence used by Butler and Cox to irradiate iron and kamacite comprised neutrons with energies of $3 \mathrm{MeV}$ or greater. It thus seems reasonable to suppose that what Butler and Cox have done in the laboratory can also be done by cosmic rays in space.

Whether these processes can account for the observed stable magnetizations in the returned lunar samples depends on two factors. The first is that the initial coercivities of iron and kamacite used in the experiments of Butler and Cox were low. Experiments on industrially important ferromagnetic materials have shown that the coercivity increase under irradiation is particularly marked when the initial coercivity is low, and it remains to be seen whether the phenomenon is also applicable to some of the minerals of higher coercive force present in lunar rocks. The second factor is, of course, that the lunar samples must have acquired an IRM in the first place. Mechanisms for this are hard to find although one possibility is the interplanetary magnetic field of 10 to 20 Gauss which Sonnet et al. (Astrophys. Space Sci., 7, 446 ; 1970) recently suggested might be the result of strong solar magnetic flares. An interesting point here is that only one such event would be required-as long as it took place subsequent to the formation of the youngest lunar rocks - so the magnetizing process need not be a particularly common phenomenon.

And there is another interesting consequence of stable magnetization by irradiation, as Butler and Cox point out. Because the parent body does not require a magnetic field, a restriction is removed from theories for the origin of meteorites. The presence of natural magnetization in chondritic meteorites has-as in lunar samples-been taken to mean that meteorites must originate on a body large enough to support, or have supported, a fluid conducting core. Many of the older meteorite theories, however, placed their origin in the asteroid belt or at least in parent bodies of asteroid size. Such bodies would not, of course, be large enough to possess an internal magnetic field. So if irradiation magnetization is possible, so apparently are asteroidal meteorites. 